\title{
Labyrinthe
}

17 | 2004 (1)

Jacques Rancière, l'indiscipliné

\section{Du possible au virtuel : la scène politique}

\section{Déborah Cohen}

\section{(2) OpenEdition}

\section{Journals}

Édition électronique

URL : http://journals.openedition.org/labyrinthe/170

DOI : 10.4000/labyrinthe. 170

ISSN : 1950-6031

Éditeur

Hermann

Édition imprimée

Date de publication : 15 avril 2004

Pagination : 27-31

Référence électronique

Déborah Cohen, « Du possible au virtuel : la scène politique », Labyrinthe [En ligne], 17 | 2004 (1), mis en ligne le 13 juin 2008, consulté le 22 avril 2019. URL : http://journals.openedition.org/labyrinthe/170 : DOI : $10.4000 /$ labyrinthe.170 


\section{DU POSSIBLE AU VIRTUEL: LA SCÈNE POLITIQUE}

Déborah CoHEN

Si Rancière a rappelé, avec La Presse d'Émile de Girardin paraissant dans les années 1840 , que "l'émancipation ouvrière ne peut être que la disparition de la classe ouvrière comme telle » $(N P, 273)$, c'est qu'un tel propos conduit ou convient à son refus de penser les classes et toute catégorie comme des essences. Mais si le marxisme confère au seul temps de l'advenir le pouvoir de défaire les catégories, c'est ici et maintenant que Rancière exige le triomphe de la lutte contre l'essentialisme. C'est ici et maintenant que doivent se creuser le vide ou se déployer l'excès qui brisent les forteresses de l'enfermement catégoriel. Excès, d'une part, qui se pose contre les procès de catégorisation, d'identification au nom d'un groupe ou d'une classe, en se projetant dans une logique de subjectivation, qui est prise du nom de ce qui est hors compte. Cette prise de nom n'est pas l'identification processuelle à un corps qui prendrait consistance dans le même mouvement, elle n'est pas prise de conscience de son essence par un groupe. Il ne peut donc s'agir de l'advenir dans le temps d'une réalité sociale qui n'était pas ou d'une conscience de l'exploitation jusque-là inaperçue: l'émancipation ouvrière ne peut s'armer des pouvoirs du temps. Vide, d'autre part, et corrélativement, ou manque de ce qu'on n'est pas, mais qu'on pourrait être; vide ou manque de cette poésie qui n'en finit pas de pointer au bout des nuits ouvrières, et qui est pourtant comme déjà là, révélant «un être voué à autre chose que l'exploitation » $(N P, 32)$. L'excès ou le manque, ce n'est pas ce qui sera ou ne sera pas; ils sont ce qui n'est pas actualisé dans le temps, mais peut être envisagé absolument et n'en possède donc pas moins une forme de présence. Penser cet excès et ce vide, lutter contre l'essentialisme, exige donc de concevoir le temps autrement, de ne pas le constituer « comme principe d'immanence subsumant tous les phénomènes sous une loi d'intériorité ${ }^{1} \gg$. Rancière 
nous convie à brouiller les frontières entre ce qui est et ce qui n'est pas, entre ce qui serait seul pensable ou faisable en une époque donnée et ce qui ne le serait pas.

Aux moroses « réalistes » de tout poil qui viendront clabauder contre, assurer que chaque époque se définit d'être une série de possibles ou d'impossibles, et refermer le temps entre les bornes supposées de ce qui est identique à soi-même, Rancière oppose le refus de l'impensable. Ce refus est certes d'abord de principe. Au passé, il est exigence en négatif: contre les arguments négationnistes, il faut dire que tout ce qui a été relève du possible et du pensable. Si tout est pensable, alors Auschwitz ne peut être nié, non seulement dans l'ordre des faits mais dans celui de la logique ${ }^{2}$. L'événement se produit en se moquant de savoir si les conditions sont réunies ou non pour ce faire, il survient quand bien même on le dirait impossible. Au présent, le refus de l'impossible est exigence en positif, contre le réalisme d'un ordre qui dresse des bornes et les déclare infranchissables, brisant toute espérance et toute action possible. Cette nécessité de considérer comme possible ce qui est dit impensable ne fixe pas un but, mais un point de départ. Ainsi de l'égalité des intelligences, qui fonctionne sur le mode du postulat, du principe $(M I)$. L'utopie (et Rancière n'aime pas le mot) ne peut alors être un lieu autre où chaque chose serait enfin à sa juste place, un ailleurs que l'on essaierait d'inscrire dans la matérialité présente ou future; elle est « ce qui fait voir la réalité présente comme non nécessaire », elle « suspend l'adhésion à un donné en proposant une question plus qu'un programme ${ }^{3} »$. Quelque chose se profile, qui n'existe pas dans l'ordre du réel des choses, dans le partage du sensible tel qu'il s'est établi, mais traverse le présent dans l'ordre du principe; son action s'inscrit ici et maintenant, sur un terrain qu'on ne peut comprendre avec les yeux d'un historicisme qui soumet le réel au possible selon le temps, ne voit d'autre mode d'existence pour le possible qu'un advenir prochain ${ }^{4}$. À ce possible identifié à l'advenir

2. Ce thème n'est pas mineur pour Rancière, qui y revient à plusieurs reprises. Voir en particulier Més., l'article précité dans L'Inactuel, et « L'inoubliable » $(A H)$. Voir encore ses réponses sur ce sujet dans l'entretien accordé à Mouvements, n 3, mars-avril 1999, p. 133-144.

3. «Sens et usage de l'utopie », dans L'Utopie en questions, 2001, p. 66.

4. Voir «Les énoncés de la fin et du rien », dans Traversée du nihilisme, 1994, p. 67-91. 


\section{Du possible au virtuel}

qu'il récuse, Rancière substitue la notion de virtuel, qui n'est pas projeté dans le temps mais existe « en surimpression » ou «pardessus » le monde donné (voir Més., 87-89). Le virtuel, ou ce que Rancière appelle aussi parfois l'apparence, est ce qui permet de casser l'opposition entre l'utopique et le réel, entre l'avenir et le présent. Il désigne une scène, un théâtre où il y a place pour ce qui était impensable selon l'ordre policier, où l'on fait «comme $s i^{5}$ ».

Rancière nous prévient : cette scène virtuelle n'est pas illusoire $(A B$, 93). Est-ce pour cela que le philosophe se fait historien? Pour nous donner par ce qui fut autant d'exemples de ce qui peut être? S'agit-il en somme de dire «par ce qui s'est fait, considérons ce qui peut se faire $^{6} »$ ? Assurément pas : les faits historiques qui ont vu surgir « l'événement égalitaire » ne sont pas pensés par Rancière comme des assurances sur l'existence de cette égalité, comme un bon lieu ou une essence un moment aperçus et à étendre. D'abord, parce que l'apparence ou le virtuel, en tant que principes, sont complets en leur genre, qui n'est pas de l'ordre du lieu. Le principe ne peut se figer en instance de réalité sans retomber dans une logique des assignations identitaires, des lieux, places et fonctions, sans recréer des essences. Il est « mis en acte », mais comme un surgissement temporaire, incomplet et fragile. Il n'abolit pas l'écart entre deux mondes, celui du présent et celui du principe qui reste ce tiers exclu, il ne fait pas basculer dans l'avenir mais reste sur ses bords. Si apparence n'est pas pour autant illusion, c'est que le virtuel modifie la figure du visible. En déployant une scène qui n'existe pas, en posant comme possible ce qui était dit impossible, en se constituant comme des êtres parlants partageant les mêmes propriétés que les patriciens ou les patrons, les plébéiens réunis sur l'Aventin ou les militants ouvriers de l'âge industriel ne constituent pas un nouveau groupe social, ils ne font pas non plus exister une égalité parfaite, mais ils agissent comme si existait, dans l'ordre du langage, ce qui n'existe pas de fait dans le partage du sensible tel qu'il se présente. Ils font donc la démonstration à la fois de l'existence de l'égalité dans le monde du principe et de son inexistence dans la cité

5. « Pour moi la politique est la constitution d'une sphère théâtrale et artificielle », dit Rancière au cours d'un entretien avec Éric Alliez, « Biopolitique ou politique? », dans Multitudes, n 1, 2000. 6. Jean -Jacques Rousseau, Du contrat social, Paris, 10/18, 1973, p. 161. 
policière. Faisant se rencontrer ces deux mondes, ils introduisent dans le champ de l'expérience un visible qui modifie le régime du visible, rompt avec les assignations traditionnelles. Cette manifestation d'un écart, d'un impensé, « fait voir une chose dans une autre » (Més., 87) et met en péril le réalisme pour qui il y a de l'impensable.

Néanmoins, l'ensemble de ce raisonnement, qui donne sens et force à ce qui est sans lieu, ne peut être validé que si l'on admet avec Rancière que le réel « doit être fictionné pour être pensé » $(P S, 61)$ et relève du discours et des symboles, tel que ce qui fait événement soit d'abord « un déplacement du dire » $(\mathrm{NH}, 65)$, une atteinte au « régime existant des rapports entre le symbolique et le réel ${ }^{7} »$. Sur l'importance du symbolique, on peut considérer dans un premier temps que Rancière se trouve d'accord avec Pierre Bourdieu pour concevoir, d'une part, que «les catégories selon lesquelles un groupe se pense et selon lesquelles il se représente sa propre réalité contribuent à la réalité de ce groupe ${ }^{8}$ » et, d'autre part, que tout ce qui se présente apparemment comme description du social n'est au fond que prescription idéologique d'un devoir-être, assignation identitaire posée par le discours dominant. Chez Rancière comme chez Bourdieu, le changement sociétal exigé est d'abord renversement ou vacillement des structures symboliques dominantes. Mais, chez Bourdieu, continue à exister un partage net entre ce qu'il appelle le « réel » et les discours symboliques - dont la force n'est, selon lui, d'autant plus grande qu' autant qu'elle est objectivement fondée. Or, c'est la validité de ce partage, entre la chose même et le discours, que remet en question Rancière: pour lui, l'ordre du symbolique, du principe, de la volonté ou du désir sont aussi « réels » que les façons de boire, de manger, et tout ce qui a pu s'inscrire dans les corps sous forme de ce que Bourdieu nomme habitus. Loin de s'ajuster à des possibles « objectifs » ou définis par un certain partage du sensible, comme chez Bourdieu', les volontés et les désirs sont vus par Rancière comme libres par rapport à leur satisfaction

7. « le 11 septembre et après : une rupture de l'ordre symbolique? », dans Lignes, $\mathrm{n}^{\circ}$ 8, 2002, p. 36. 8. Pierre Bourdieu, « Décrire et prescrire: les conditions de possibilité et les limites de l'efficacité politique », dans Langage et pouvoir symbolique, Paris, Fayard, 2001, p. 195.

9. Voir par exemple ses Méditations pascaliennes, chap. 6 « L'être social, le temps et le sens de l'existence », Paris, Seuil, coll. « Liber», 1997. 
promise. Tandis que le présent bourdieusien est envahi par la présence de «l'à venir », de ce qui, étant vu comme inéluctable, limite les investissements, les rêves et les façons d'agir, le présent de Rancière est peuplé de virtualités, d'un futur qui modifie déjà le présent - à condition que l'on croie encore au pouvoir des mots et des symboles. C'est sur le désir commun aux hommes des jouissances de la parole et de la pensée que parie Rancière pour fonder la réalité du virtuel. 